\title{
Aspidosperma Species as Sources of Antimalarials. Part III. A Review of Traditional Use and Antimalarial Activity*
}

Authors

Affiliations
Renata Cristina de Paula ${ }^{1 * *}$, Maria Fâni Dolabela ${ }^{2 * *}$, Alaíde Braga de Oliveira ${ }^{1 * *}$

${ }^{1}$ Departamento de Produtos Farmacêuticos, Faculdade de Farmácia, Universidade Federal de Minas Gerais, Belo Horizonte, MG, Brazil

${ }^{2}$ Departamento de Farmácia, ICS, Universidade Federal do Pará, Belém, PA, Brazil
Key words

- Aspidosperma spp.

- Apocynaceae

- malaria

- Plasmodium falciparum

- antimalarial activity received August 18, 2013 revised January 10, 2014 accepted January 11, 2014

\section{Bibliography}

Dol http://dx.doi.org/

10.1055/s-0034-1368168

Published online March 3, 2014

Planta Med 2014; 80: 378-386

(c) Georg Thieme Verlag KG

Stuttgart · New York .

ISSN 0032-0943

\section{Correspondence}

\section{Alaíde Braga de Oliveira}

Departamento de Produtos

Farmacêuticos

Faculdade de Farmácia

Universidade Federal de Minas

Gerais

Av. Antônio Carlos, 6.627

31270-901 Belo Horizonte, MG Brazil

Phone: + 553134096950

Fax: + 553134415575

alaide.braga@pq.cnpq.br

\section{Abstract \\ $\nabla$}

Several plant species belonging to the genus Aspidosperma are traditionally used in Brazil and other Meso- and South American countries for the treatment of malaria and fevers. These traditional uses were motivation for this review. A literature survey completed for this review has identified scientific bibliographical references to the use of 24 Aspidosperma species to treat malaria/fevers and to 19 species that have had their extracts

\section{Introduction}

$\nabla$

\section{Natural products and malaria}

Malaria remains one of the most prevalent infectious diseases worldwide and is, therefore, a global health problem despite substantial efforts to control the disease over the past few decades. Approximately 3.3 billion people are at risk, and 250 million cases each year were reported in the period 2006-2008, primarily in Africa [1]. In the Americas, malaria transmission occurs in 21 countries. P. vivax caused $77 \%$ of all cases reported in 2008 , but $P$. falciparum was responsible for almost $100 \%$ of all cases in Haiti and the Dominican Republic [1]. Brazil reported the highest number of malaria cases (603 532) in the region in 2005, primarily in the Brazilian Legal Amazon Region, where $10-15 \%$ of the population is at risk. Brazil was among the 30 highest-burden countries for malaria [2]. However, a decrease of approximately $25 \%$ in the number of reported cases has been recorded since $2006[3,4]$.

Historically, plants have had a remarkable role in therapeutics and were the principal source of drugs until the 19th century. Quinine, isolated in 1820, from Cinchona species (Rubiaceae), was the

\footnotetext{
* For Part I see [48], for Part II see [36]. Part of RCP Doctorate Thesis at PPGCF, UFMG, Belo Horizonte, MG, Brazil.
}

and/or alkaloids evaluated, with good results, for in vitro and/or in vivo antimalarial activity. Indole alkaloids are typical constituents of Aspidosperma species. However, only 20 out of more than 200 known indole alkaloids isolated from this genus have been assayed for antimalarial activity. These data support the potential of Aspidosperma species as sources of antimalarials and the importance of research aimed at validating their use in the treatment of human malaria. first antimalarial drug introduced in chemotherapy and remained the only clinical weapon until the 1940s, when chloroquine, a synthetic 4-aminoquinoline, became available. Efficient and inexpensive, chloroquine was widely used until the 1960s, when resistance to the drug by $P$. falciparum became widespread in the malaria-endemic countries, causing a strong increase in mortality rates. The antimalarial drugs in current use are artemisinin, the active compound from Artemisia annua L. (Asteraceae), a traditional plant used for millennia in China, and its semisynthetic derivatives artemether, artesunate, and arteether [5]. Artemisinins are currently the most effective drugs for antimalarial chemotherapy and have been globally adopted for the treatment of $P$. falciparum malaria. The most recently introduced antimalarial drug is atovaquone, a synthetic naphthoquinone based on lapachol. Lapachol, a prenylnaphtoquinone, was first isolated from $\mathrm{Ta}$ bebuia impetiginosa (Mart. ex DC.) Standl. (synon. T. avellanedeae Lor. ex Griseb.), a South American representative of the Bignoniaceae [6].

The emergence of $P$. falciparum strains resistant to artemisinin and its derivatives would cause a resurgence of human malaria to high levels in many

** All authors contributed equally to this article. 
countries. For this reason, R\&D on new antimalarial drugs is urgent. Plants continue to represent a valuable source of drugs. A review of all small molecules that have been approved as pharmaceutical agents within the 25-year period from 1/1981 to 6/ 2006 has demonstrated that approximately $50 \%$ of these molecules originated from natural products [7]. Investigations that begin by screening plants used in traditional medicines are particularly valuable. In the past decade, a substantial number of publications have focused on the screening of either extracts or natural products for antimalarial activity [8]. However, as we have noted previously, very few highly active antimalarial natural products have been evaluated for cytotoxicity and in in vivo assays. This lack of previous study limits the potential of these products as bases for the development of new antimalarial drugs $[5,9]$. The relevance of these results should not, however, be underestimated. Active naturally occurring antimalarial compounds might be useful as bases for semisynthetic derivatives, as agents for direct use if their structures are too complex for an economically and/or technologically viable total synthesis, or as templates for the total synthesis of structurally related compounds [5]. Moreover, bioactive natural products from medicinal plants might be useful as biological markers in the development of efficient and safe phytomedicines [5,9], a new approach of growing interest that would provide malaria-endemic countries with good-quality herbal medicines of low cost, that would be locally and sustainably produced [8]. High-tech methods are available to standardize phytopreparations [10,11], and new molecular biological assays can serve to screen extracts and plant constituents as well as to evaluate their pharmacological profiles, elucidate the synergistic effects of the constituents of an extract and, thus, gain a better understanding of the various mechanisms underlying their pharmacological effects [11-14].

Several Aspidosperma species have a history of medicinal uses, including the treatment of human malaria and/or fevers in Brazil as well as in other Meso- and South American countries [15-19]. The present review reports the results of a literature survey on Aspidosperma species traditionally used to treat malaria and/or fevers as well as data on those previously evaluated for antimalarial activity.

\section{The Genus Aspidosperma}

$\nabla$

\section{Taxonomy and geographic distribution}

The genus Aspidosperma Mart. \& Zucc. belongs to the family Apocynaceae, one of the ten largest angiosperm families [20]. The Apocynaceae s.l. belong to the order Gentianales and have a primarily pantropical/subtropical distribution with few temperate representatives [21].

Aspidosperma species are of outstanding economic interest as sources of valuable timber. They are found from Mexico to Argentina. The number of species in this genus is controversial. Woodson [22] recognizes 52 species, whereas Marcondes-Ferreira and Kinoshita [23] have proposed an infra-generic division for this genus with 43 species, 32 of them occurring in Brazil. However, in the "Lista de Espécies da Flora do Brasil (LEFB)", 51 species are listed with accepted names; 21 of these species are endemic to Brazil, and 90 synonyms are reported [24].

\section{Traditional use in treating malaria}

Data obtained by searching for Aspidosperma species with reported traditional uses as antimalarials and/or febrifuges as well as those species that have been experimentally evaluated for antimalarial activity are shown in $\odot$ Table $\mathbf{1}$. The table includes the accepted taxonomic nomenclature, synonyms, and occurrence, according to Koch and collaborators [20], LEFB [24], Lorenzi (1992) [15], TROPICOS ${ }^{\circledR}$ Specimen Data Base [25], and The Plant List [26]. The reported uses for treating malaria/fevers are based on the scientific literature. Medicinal uses described on commercial Internet sites, as well as uses other than for malaria and/or fevers, have not been included. Reported antimalarial activity may refer to in vitro assays with different Plasmodium species, in vivo assays in different animals with different Plasmodium species, or clinical assays in humans. Details of these findings are shown in 0 Table 2.

Our literature survey has identified 24 Aspidosperma species ( $O$ Table 1) used to treat malaria/fevers, representing approximately $50 \%$ of the representatives of this taxon [24]. In addition to these 24 species, $\bigcirc$ Table 1 includes two other species, A. cylindrocapon and A. macrocarpon, for which no reports have been found on their use to treat malaria/fever but that have been evaluated for antimalarial activity $[27,28]$.

The "Dicionário das plantas úteis do Brasil e das exóticas cultivadas" (Dictionary of useful plants from Brazil and of the exotics cultivated), by Pio-Corrêa (1874-1934) [17], was our first source of information on the antimalarial use of plants belonging to the genus Aspidosperma. More than 50 Aspidosperma species are listed within this six-volume collection, with taxonomic data and vernacular names. Most of the applications described there are as sources of timber, whereas only three species, A. discolor, A. polyneuron, and A. gomezianum, are identified as plants used to treat malaria and/or fevers.

The botanical names shown for the 26 Aspidosperma species in - Table 1 are those originally reported in the literature. However, it should be emphasized that these are not the presently accepted names in certain cases. This is the situation for A. marcgravianum Woodson (A. marckgravianum), which is not presently an accepted name [24] but a synonym of $A$. excelsum Benth. together with A. nitidum Benth. ex Müll. Arg., according to Koch and collaborators [20] and LEFB [24], but is described as a synonym for A. excelsum only by the The Plant List [26]. In the same source [26], however, A. nitidum Benth. ex Müll. Arg. is an accepted name, with $A$. acquaticum Ducke as a synonym. Another contradiction is found for A. album (Vahl) Benoist ex Pichon and A. desmanthum Benth. ex Müll. Arg., cited as accepted names for distinct species $[20,24,26]$, whereas the second species represents a synonym for the first one [25]. Furthermore, A. parvifolium A. DC. is the accepted name of a species that has A. tambopatense A.H. Gentry and A. vargasii A. DC. as synonyms [20,24], whereas the latter synonym is also cited as a distinct species $[25,26]$. A similar situation is observed for A. tomentosum Mart., an accepted name having A. gomezianum A. DC. as a synonym [25, 26], although both of them are also reported as distinct species $[20,26]$. These observations show that several controversies remain in the taxonomy of Aspidosperma species.

\section{Phytochemistry and antimalarial activity}

Chemically, Aspidosperma species are characterized by the presence of alkaloids and have yielded more than 200 indole alkaloids. The phytochemical investigation of several Brazilian Aspidosperma species, primarily in the period between 1960 and 
Table 1 Literature survey on Aspidosperma species, their traditional use to treat malaria and/or fevers, and reported antimalarial activity.

\begin{tabular}{|c|c|c|c|c|}
\hline Aspidosperma species & $\begin{array}{l}\text { Local names in Brazil } \\
\text { References: }[15,17 \text {, } \\
20,24]\end{array}$ & $\begin{array}{l}\text { Occurrence } \\
\text { References: }[15,17,20,24-26]\end{array}$ & $\begin{array}{l}\text { Reported use to } \\
\text { treat malaria/ } \\
\text { fever }\end{array}$ & $\begin{array}{l}\text { Reported anti- } \\
\text { malarial activity }\end{array}$ \\
\hline $\begin{array}{l}\text { A. album (Vahl) Benoist ex Pichon } \\
\text { Syn. A. desmanthum Benth. ex Müll. Arg., } \\
\text { A. centrale Markg. }[20,24,26]\end{array}$ & $\begin{array}{l}\text { Cabeça-de-arara, Piquiá- } \\
\text { marfim, Araracanga, } \\
\text { Aracanga-preta, Mapa- } \\
\text { rana }\end{array}$ & $\begin{array}{l}\text { Bolívia, Colômbia, French Guiana, } \\
\text { Guyana, Suriname, Venezuela, } \\
\text { Brazil: Amazonia }\end{array}$ & $\begin{array}{l}\text { Yes } \\
{[62,63]}\end{array}$ & No \\
\hline A. auriculatum Markgr. $[20,26]$ & $\begin{array}{l}\text { Carapanaúba } \\
\text { Carapanaúba-amarela }\end{array}$ & Brazil: Endemic (PA) & $\begin{array}{l}\text { Yes } \\
{[64]}\end{array}$ & No \\
\hline $\begin{array}{l}\text { A. cuspa (Kunth) S. F. Blake ex Pittier } \\
\text { Syn. A. decipiens Müll. Arg. } \\
\text { (+ } 11 \text { synon.) }[20,26]\end{array}$ & $\begin{array}{l}\text { Guatambuzinho, } \\
\text { Guatambú-branco, } \\
\text { Amargoso, Peroba-de- } \\
\text { Goiás }\end{array}$ & $\begin{array}{l}\text { Bolivia, Colombia, Ecuador, Haiti, Vene- } \\
\text { zuela, Paraguay, Peru, Republica Domini- } \\
\text { cana, Trinidad Tobago, Venezuela, Brazil: } \\
\text { Amazonia, Caatinga, Cerrado, Atlantic } \\
\text { Rainforest }\end{array}$ & $\begin{array}{l}\text { Yes } \\
{[65]}\end{array}$ & No \\
\hline $\begin{array}{l}\text { A. cylindrocarpon Müll. Arg. } \\
\text { Syn. A. brevifolia Rusby [20,26] }\end{array}$ & $\begin{array}{l}\text { Peroba-iquira, Peroba de } \\
\text { Lagoa Santa, Peroba de } \\
\text { Minas, Peroba rosa }\end{array}$ & $\begin{array}{l}\text { Bolivia, Paraguay Peru, Brazil: Amazônia, } \\
\text { Cerrado, Atlantic Rainforest }\end{array}$ & No & $\begin{array}{l}\text { Yes } \\
\text { See } \bullet \text { Table } 2\end{array}$ \\
\hline $\begin{array}{l}\text { A. desmanthum Benth. ex Müll. Arg } \\
\text { Accepted name } \\
\text { Syn. A. cruentum Woodson, A. chiapense } \\
\text { Matuda, A. matudae Lundell }[20,24,26] \\
\text { Syn. A. album (Vahl) Benoist ex Pichon } \\
\text { [25] }\end{array}$ & $\begin{array}{l}\text { Araraíba, Peroba, Arrara- } \\
\text { canga, Amargoso, } \\
\text { Pequiá-marfim }\end{array}$ & $\begin{array}{l}\text { Belize, Bolivia, Colombia, Ecuador, French } \\
\text { Guiana, Honduras, Mexico, Panama, } \\
\text { Nicaragua, Brazil: Amazonia, Atlantic } \\
\text { Forest }\end{array}$ & $\begin{array}{l}\text { Yes } \\
{[17,63,66]}\end{array}$ & $\begin{array}{l}\text { Yes } \\
\text { See } \bigcirc \text { Table } 2\end{array}$ \\
\hline $\begin{array}{l}\text { A. discolor A. DC. } \\
\text { Accepted name } \\
\text { Syn. A. francisii A. DC. }[20,26]\end{array}$ & $\begin{array}{l}\text { Cabo-de-machado, } \\
\text { Quina, Carapanaúba- } \\
\text { amarela, Pau-pereira, } \\
\text { Peroba-de-rego, Peroba }\end{array}$ & $\begin{array}{l}\text { Bolívia, French Guiana, Guyana, Peru, } \\
\text { Suriname, Venezuela, Brazil: Amazonia, } \\
\text { Caatinga, Cerrado, Atlantic Forest }\end{array}$ & $\begin{array}{l}\text { Yes } \\
{[17]}\end{array}$ & No \\
\hline $\begin{array}{l}\text { A. excelsum Benth. } \\
\text { Accepted name } \\
\text { Syn. A. marcgravianum Woodson, A. niti- } \\
\text { dum Benth. ex Müll. Arg. }[20,25,26]\end{array}$ & $\begin{array}{l}\text { Carapanaúba, } \\
\text { Carapanaúba-preta, } \\
\text { Sapopema, Sapopemba }\end{array}$ & $\begin{array}{l}\text { Costa Rica, Panama, Colombia, French } \\
\text { Guiana, Guyana, Ecuador, Peru, Bolivia, } \\
\text { Venezuela, Brazil: N, NE, SE, CW }\end{array}$ & $\begin{array}{l}\text { Yes } \\
{[67-70]}\end{array}$ & $\begin{array}{l}\text { Yes } \\
\text { See } 0 \text { Table } 2\end{array}$ \\
\hline $\begin{array}{l}\text { A. gomezianum A. DC. } \\
\text { Accepted name }[20,24] \\
\text { Non-cons., syn. of A. tomentosum Mart. } \\
{[25]}\end{array}$ & $\begin{array}{l}\text { Peroba-amarela, Pequiá- } \\
\text { de-pedra, Pau-cetim, } \\
\text { Guatambu, Ipê-peroba }\end{array}$ & $\begin{array}{l}\text { Brazil: NE (Bahia), SE/Minas Gerais, } \\
\text { Espirito Santo, Rio de Janeiro }\end{array}$ & $\begin{array}{l}\text { Yes } \\
{[17,28]}\end{array}$ & No \\
\hline $\begin{array}{l}\text { A. macrocarpon Mart. } \\
\text { Accepted name } \\
\text { Syn. A. macrocarpum Mart., A. duckei } \\
\text { Huber ex Ducke, A. gardneri Müll. Arg. } \\
\text { (+ } 6 \text { other syn.) }[20,26]\end{array}$ & $\begin{array}{l}\text { Guatambú, Guatambú- } \\
\text { do-cerrado, Peroba-do- } \\
\text { campo, Pau-pereira, } \\
\text { Pereiro }\end{array}$ & $\begin{array}{l}\text { Bolívia, Ecuador, Peru, Brazil: Amazonia, } \\
\text { Cerrado }\end{array}$ & No & $\begin{array}{l}\text { Yes } \\
\text { See } \bullet \text { Table } 2\end{array}$ \\
\hline $\begin{array}{l}\text { A. marcgravianum Woodson } \\
\text { This name is a synonym of } A \text {. excelsum } \\
\text { Benth and A. nitidum Benth. ex Müll. Arg. } \\
{[24,26]}\end{array}$ & $\begin{array}{l}\text { Carapanaúba, Carana- } \\
\text { paúba-preta }\end{array}$ & $\begin{array}{l}\text { Bolivia, Colômbia, French Guiana, } \\
\text { Guyana, Ecuador, Panamá, Peru, } \\
\text { Suriname, Venezuela }\end{array}$ & $\begin{array}{l}\text { Yes } \\
{[71]}\end{array}$ & $\begin{array}{l}\text { Yes } \\
\text { See } \oslash \text { Table } 2\end{array}$ \\
\hline $\begin{array}{l}\text { A. megalocarpon Müll. Arg. } \\
\text { Accepted name } \\
\text { Syn. A. lundellianum Woodson, } \\
\text { A. saguinale Bartl., } \\
\text { A. stegomeris (Woodson) Woodson [22, } \\
26]\end{array}$ & $\begin{array}{l}\text { Pequiá-marfim, Arara- } \\
\text { canga, Carapanaúba, } \\
\text { Carapanaúba-preta }\end{array}$ & $\begin{array}{l}\text { Central America from México to } \\
\text { Colombia [25], Brazil: Endemic (Acre) } \\
\text { [24] }\end{array}$ & $\begin{array}{l}\text { Yes } \\
{[36]}\end{array}$ & $\begin{array}{l}\text { Yes } \\
\text { See } \odot \text { Table } 2\end{array}$ \\
\hline $\begin{array}{l}\text { A. nitidum Benth ex Müll. Arg. } \\
\text { Accepted name } \\
\text { Syn. A. aquaticum Ducke, } \\
\text { Syn. of A. excelsum Benth. [24-26] }\end{array}$ & Carapanaúba, Maparaná & $\begin{array}{l}\text { Peru [25], Brazil: Amazonas [25], } \\
\text { Roraima [24] }\end{array}$ & $\begin{array}{l}\text { Yes } \\
{[72]}\end{array}$ & $\begin{array}{l}\text { Yes } \\
\text { See } \odot \text { Table } 2\end{array}$ \\
\hline $\begin{array}{l}\text { A. oblongum A. DC. } \\
\text { Accepted name } \\
\text { Syn. A. khulmannii Markgr. [26] }\end{array}$ & $\begin{array}{l}\text { Carapanaúba, Carapa- } \\
\text { naúba- amarela }\end{array}$ & $\begin{array}{l}\text { French Guiana, Guyana, Suriname, } \\
\text { Venezuela, Brazil: Pará, Amazonas, } \\
\text { Maranhão, Goiás }\end{array}$ & $\begin{array}{l}\text { Yes } \\
{[73]} \\
\text { (A. oblongum) }\end{array}$ & $\begin{array}{l}\text { Yes } \\
\text { See } 0 \text { Table } 2\end{array}$ \\
\hline $\begin{array}{l}\text { A. olivaceum Müll. Arg. } \\
\text { Accepted name } \\
\text { Syn. A. bello-horizontinum Silveira [20,26] }\end{array}$ & $\begin{array}{l}\text { Guatambú, Guatambu- } \\
\text { mirim }\end{array}$ & $\begin{array}{l}\text { Brazil: Endemic to Atlantic Rainforest } \\
\text { (NE, SE, S) [24], Argentina }[23,25]\end{array}$ & $\begin{array}{l}\text { Yes } \\
{[67]}\end{array}$ & $\begin{array}{l}\text { Yes } \\
\text { See } 0 \text { Table } 2\end{array}$ \\
\hline $\begin{array}{l}\text { A. parvifolium A. DC. } \\
\text { Accepted name } \\
\text { Syn. A. ingratum K. Schum., A. vargasii A. } \\
\text { DC., }\end{array}$ & $\begin{array}{l}\text { Pau-Pereira, Peroba, } \\
\text { Guatambú, Guatambú- } \\
\text { rosa, Guatambú-marfim } \\
\text { Amarelão }\end{array}$ & $\begin{array}{l}\text { Argentina, Bolivia,Guyana, Paraguay, } \\
\text { Peru, Venezuela, Brazil: Amazonia, } \\
\text { Caatinga, Cerrado, Atlantic Rainforest }\end{array}$ & $\begin{array}{l}\text { Yes } \\
{[74]}\end{array}$ & $\begin{array}{l}\text { Yes } \\
\text { See } \odot \text { Table } 2\end{array}$ \\
\hline
\end{tabular}

A. tambopatense A. H. Gentry $[20,25,26]$ 
Table 1 Continued

\begin{tabular}{|c|c|c|c|c|}
\hline Aspidosperma species & $\begin{array}{l}\text { Local names in Brazil } \\
\text { References: }[15,17 \text {, } \\
20,24]\end{array}$ & $\begin{array}{l}\text { Occurrence } \\
\text { References: }[15,17,20,24-26]\end{array}$ & $\begin{array}{l}\text { Reported use to } \\
\text { treat malarial } \\
\text { fever }\end{array}$ & $\begin{array}{l}\text { Reported anti- } \\
\text { malarial activity }\end{array}$ \\
\hline $\begin{array}{l}\text { A. polyneuron Müll. Arg. } \\
\text { Accepted name } \\
\text { Syn. A. dugandii Standl., A. peroba } \\
\text { Allemão ex Saldanha, } \\
\text { A. venosum Müll. Arg. }[20,24,26]\end{array}$ & $\begin{array}{l}\text { Peroba-rosa, Sobro, } \\
\text { Peroba-amargosa }\end{array}$ & $\begin{array}{l}\text { Argentina, Bolívia, Colombia, Paraguay, } \\
\text { Peru, Venezuela, Brazil: Caatinga, } \\
\text { Cerrado, Atlantic Rainforest }\end{array}$ & $\begin{array}{l}\text { Yes } \\
{[17,75]}\end{array}$ & $\begin{array}{l}\text { Yes } \\
\text { See } \bullet \text { Table } 2\end{array}$ \\
\hline $\begin{array}{l}\text { A. pyrifolium Mart. } \\
\text { Accepted name } \\
\text { Syn. A. bicolor Mart. (+ several others) } \\
\text { [26] }\end{array}$ & $\begin{array}{l}\text { Pereiro-do-sertão, } \\
\text { Pereiro-preto, Pau-de- } \\
\text { coam, Pequiá-da-rest- } \\
\text { inga }\end{array}$ & $\begin{array}{l}\text { Argentina, Bolívia, Paraguay, } \\
\text { Brazil: Caatinga, Cerrado }\end{array}$ & $\begin{array}{l}\text { Yes } \\
{[62]}\end{array}$ & $\begin{array}{l}\text { Yes } \\
\text { See } \bullet \text { Table } 2\end{array}$ \\
\hline $\begin{array}{l}\text { A. quebracho blanco Schltdl. } \\
\text { Accepted name } \\
\text { Syn. A. quebracho Griseb (+ several } \\
\text { others) [26] }\end{array}$ & $\begin{array}{l}\text { Quebracho-branco } \\
\text { (Brazil), Quebracho- } \\
\text { blanco (Argentina) }\end{array}$ & $\begin{array}{l}\text { Argentina, Bolivia, Paraguay, Uruguay, } \\
\text { Brazil: Mato Grosso }\end{array}$ & $\begin{array}{l}\text { Yes } \\
{[68,72,76,77]}\end{array}$ & $\begin{array}{l}\text { Yes } \\
\text { See } 0 \text { Table } 2\end{array}$ \\
\hline $\begin{array}{l}\text { A. ramiflorum Müll. Arg. } \\
\text { Accepted name } \\
\text { Syn. Geissospermum ramiflorum (Müll. } \\
\text { Arg.) Miers [26] }\end{array}$ & $\begin{array}{l}\text { Pau-pereira, Peroba, } \\
\text { Guatambú-amarelo }\end{array}$ & $\begin{array}{l}\text { Bolívia, Brazil: Amazonia } \\
\text { Cerrado, Atlantic Rainforest }\end{array}$ & $\begin{array}{l}\text { Yes } \\
{[22]}\end{array}$ & $\begin{array}{l}\text { Yes } \\
\text { See } \bullet \text { Table } 2\end{array}$ \\
\hline $\begin{array}{l}\text { A. rigidum Rusby } \\
\text { Accepted name } \\
\text { Syn. A. acreanum Markg. (+ several } \\
\text { others) }[20,26]\end{array}$ & $\begin{array}{l}\text { Carapanaúba, } \\
\text { Aracaranga }\end{array}$ & $\begin{array}{l}\text { Bolívia, Colombia, Costa Rica, Ecuador, } \\
\text { Panamá, Peru, Venezuela [25], Brazil: } \\
\text { Endemic (Amazonia, Cerrado) [24] }\end{array}$ & $\begin{array}{l}\text { Yes } \\
{[39]}\end{array}$ & $\begin{array}{l}\text { Yes } \\
\text { See } \bullet \text { Table } 2\end{array}$ \\
\hline $\begin{array}{l}\text { A. sandwithianum Markgr. } \\
\text { Accepted name } \\
\text { No synonyms }[20,26]\end{array}$ & Carapanaúba & $\begin{array}{l}\text { French Guiana, Guyana, Suriname, } \\
\text { Brazil: Pará, Amazonas }\end{array}$ & $\begin{array}{l}\text { Yes } \\
{[71]}\end{array}$ & No \\
\hline $\begin{array}{l}\text { A. schultesii Woodson } \\
\text { Accepted name } \\
\text { No synonyms }[20,26]\end{array}$ & Maku & $\begin{array}{l}\text { French Guiana, Peru, Venezuela } \\
\text { Brazil: Amazonas, Roraima, Mato Grosso }\end{array}$ & $\begin{array}{l}\text { Yes } \\
{[66,71]}\end{array}$ & No \\
\hline $\begin{array}{l}\text { A. spruceanum Benth. ex Müll. Arg. } \\
\text { Accepted name } \\
\text { Syn. A. igapoanum Markgr., } \\
\text { A. melanocalix Müll. Arg. (+ several } \\
\text { others) }[20,26]\end{array}$ & $\begin{array}{l}\text { Amargoso, Araracanga, } \\
\text { Guatambu, Peroba, } \\
\text { Quina da mata, Pequiá } \\
\text { marfim }\end{array}$ & $\begin{array}{l}\text { Bolivia, Colombia, Ecuador, French } \\
\text { Guiana, Mexico, Panama, Peru, Venezu- } \\
\text { ela, Brazil: Amazonia, Cerrado, Atlantic } \\
\text { Rainforest }\end{array}$ & $\begin{array}{l}\text { Yes } \\
{[78]}\end{array}$ & $\begin{array}{l}\text { Yes } \\
\text { See } \odot \text { Table } 2\end{array}$ \\
\hline $\begin{array}{l}\text { A. tomentosum Mart } \\
\text { Accepted name } \\
\text { Syn. A. camporum Müll. Arg., } \\
\text { A. dasycarpon A. DC. and others [20, 24, } \\
\text { 26] } \\
\text { Non-accepted name, syn. for } \\
\text { A. gomezianum A. DC. fide Woodson, Jr. } \\
\text { R. E., } 1951 \text { [25] }\end{array}$ & $\begin{array}{l}\text { Guatambú-do-cerrado, } \\
\text { Pereiro-do-campo }\end{array}$ & $\begin{array}{l}\text { Bolívia, Paraguay, Brazil: Amazonia, } \\
\text { Caatinga, Cerrado }\end{array}$ & $\begin{array}{l}\text { Yes } \\
{[79]}\end{array}$ & No \\
\hline $\begin{array}{l}\text { A. ulei Markg. } \\
\text { Accepted name } \\
\text { Syn. A. occidentale Markg. }[20,26]\end{array}$ & Pitiá & $\begin{array}{l}\text { Guyana, Suriname, Venezuela, Brasil: } \\
\text { Amazonia, Atlantic Forest }\end{array}$ & $\begin{array}{l}\text { Yes } \\
{[22]}\end{array}$ & $\begin{array}{l}\text { Yes } \\
\text { See } 0 \text { Table } 2\end{array}$ \\
\hline $\begin{array}{l}\text { A. vargasii A. DC. } \\
\text { Accepted name }[25,26] \\
\text { Non-accepted name, syn. for } \\
\text { A. parvifolium A. DC. }[20,24]\end{array}$ & Amarelão & $\begin{array}{l}\text { Bolívia, Colombia, Guyana, Peru, } \\
\text { Suriname, Venezuela }\end{array}$ & $\begin{array}{l}\text { Yes } \\
{[62,66,71]}\end{array}$ & $\begin{array}{l}\text { Yes } \\
\text { See } 0 \text { Table } 2\end{array}$ \\
\hline
\end{tabular}

1980 , is highly valuable and can be appreciated in a 2007 review [29]. However, there are relatively few reports on the biological activities of these alkaloids $[5,30]$.

Of the 26 Aspidosperma species included in Table 1, 19 have had their crude extracts and/or alkaloidal extracts assayed for antimalarial activity. Detailed data are shown in 0 Table 2. Evaluations were performed as early as 1932, and several experiments were conducted before the methodology for the $P$. falciparum erythrocytic culture became available [31]. These experiments include in vivo assays in ducklings infected with P. lophurae [32], in vitro assays with $P$. cathemerium [33], and clinical assays in humans [34,35] ( Table 2). Good activity $\left(\mathrm{IC}_{50}<10 \mu \mathrm{g} / \mathrm{mL}\right.$ ) has been reported for extracts from A. macrocarpon [28], A. megalo- carpon [36], A. oblongum [37], A. olivaceum [27], A. quebracho blanco [38], and $A$. rigidum [39]. A hydroethanolic extract from A. pyrifolium stem bark was inactive against the $P$. falciparum F32 strain [40]. However, a series of indole alkaloids isolated from the same species were shown to be active against $P$. falciparum FcM29 and Nigerian strains [41,42]. Most likely, this controversy can be explained by a low content of alkaloids in the inactive crude extract. Extracts from $A$. cylindrocarpon $[27,43]$ and A. macrocarpon [28], the two species with no reported uses in treating malaria, have shown in vitro activity against three different $P$. falciparum clones (W2, 3D7, FcB1), with a selectivity index $>10\left(\mathrm{SI}=\mathrm{CC}_{50} / \mathrm{IC}_{50}\right)$ in every case $(\bullet$ Table 2$)$. These results demonstrate the validity of the taxonomic approach for the selection 
Table 2 Antimalarial activity of Aspidosperma extracts and alkaloids.

\begin{tabular}{|c|c|c|c|c|c|c|}
\hline $\begin{array}{l}\text { Aspidosperma } \\
\text { species }\end{array}$ & $\begin{array}{l}\text { Country of } \\
\text { collection }\end{array}$ & $\begin{array}{l}\text { Part of the } \\
\text { plant }\end{array}$ & $\begin{array}{l}\text { Extracts } \\
\text { Compounds }\end{array}$ & $\begin{array}{l}\text { Bioassay } \\
\text { Plasmodium species| } \\
\text { strains }\end{array}$ & Results & $\begin{array}{l}\text { Refer- } \\
\text { ences }\end{array}$ \\
\hline A. cylindrocarpon & Brazil & Trunkwood & $\mathrm{EtOH}$ & $\begin{array}{l}\text { In vitro/P. falciparum } \\
\text { W2 and 3D7 strains }\end{array}$ & $\begin{array}{l}W 2: I_{50}=44 \mu \mathrm{g} / \mathrm{mL} \\
\text { 3D7: IC } 50=39 \mu \mathrm{g} / \mathrm{mL} \\
\text { Cytotoxicity } \\
\text { Vero cells } C_{50}>500 \mu \mathrm{g} / \mathrm{mL} \\
\text { SI: } W 2=11.4 \\
\text { SI: } 3 D 7=12.8\end{array}$ & [27] \\
\hline A. desmanthum & Brazil & Trunk bark & $\begin{array}{l}\text { Aspidocarpine } \\
\text { (2) }\end{array}$ & In vitro/P. falciparum K1 & $\mathrm{IC}_{50}=0.019 \mu \mathrm{M}(0.07 \mu \mathrm{g} / \mathrm{mL})$ & [45] \\
\hline A. excelsum & $\begin{array}{l}\text { Peru } \\
\text { (Remo cas- } \\
\text { pi) }\end{array}$ & Trunk bark & $\mathrm{EtOH}$ & $\begin{array}{l}\text { In vitro/P. falciparum } \\
\text { 3D7 }\end{array}$ & $\begin{array}{l}\mathrm{IC}_{50}=42 \mu \mathrm{g} / \mathrm{mL} \\
\mathrm{SI} \text { human lymphocyte inhibition } \\
\mathrm{IC}_{50}>100 \mu \mathrm{g} / \mathrm{mL}\end{array}$ & [39] \\
\hline A. macrocarpon & Brazil & Root bark & $\mathrm{EtOH}$ & $\begin{array}{l}\text { In vitro/P. falciparum } \\
\text { FCB1 }\end{array}$ & $\begin{array}{l}\mathrm{FcB1}: \mathrm{IC} \mathrm{C}_{50}=4.9 \mu \mathrm{g} / \mathrm{mL} \\
\text { Cytotoxicity MRC- } 5 \mathrm{CC}_{50}=79.2 \mu \mathrm{g} / \mathrm{mL} \\
\mathrm{SI}=16.2\end{array}$ & [28] \\
\hline $\begin{array}{l}\text { A. marcgravia- } \\
\text { num }\end{array}$ & Guyane & Leaves & $\begin{array}{l}\text { Tetrahydro- } \\
\text { usambaresine } \\
\text { (17S) }\end{array}$ & $\begin{array}{l}\text { In vitro/P. falciparum } \\
\text { FcM29 }\end{array}$ & $0.26 \mu \mathrm{g} / \mathrm{mL}(0.59 \mu \mathrm{M})$ & [32] \\
\hline A. megalocarpon & Colombia & Trunk bark & $\mathrm{MeOH}$ & $\begin{array}{l}\text { In vitro/P. falciparum } \\
\text { D2 and F32 strains }\end{array}$ & $\begin{array}{l}\text { MeOH extract } \\
\text { D2 strain: } \mathrm{IC}_{50}=8 \mu \mathrm{g} / \mathrm{mL} \\
\text { F32 strain: } \mathrm{IC}_{50}=25 \mu \mathrm{gg} / \mathrm{mL} \\
\text { Cytotoxicity U-937 human promonocitic } \\
\text { cells: } \mathrm{CC}_{50}=0.4 \mu \mathrm{g} / \mathrm{mL} \\
\text { SI: Pf } / \mathrm{D} 2=0.05 \\
\text { SI: Pf/F32 }=0.02\end{array}$ & [36] \\
\hline A. megalocarpon & Colombia & & $\begin{array}{l}\text { Fendlerine (13), } \\
\text { Aspidoalbine } \\
\text { (14), Aspidolimi- } \\
\text { dine (15) }\end{array}$ & $\begin{array}{l}\text { In vitro/P. falciparum } \\
\text { FcM29 and Nigerian } \\
\text { strains }\end{array}$ & $\begin{array}{l}\text { FcM29: } \mathrm{IC}_{50}=25.6 \text { to } 59.2 \mu \mathrm{g} / \mathrm{mL} \\
\text { Nigerian strain: } \mathrm{I} C_{50}=28.0 \text { to } 57.3 \mu \mathrm{g} / \mathrm{mL}\end{array}$ & {$[41,53]$} \\
\hline A. nitidum & Brazil & - & - & $\begin{array}{l}\text { In vivo } \\
\text { Duckling/P. lophurae }\end{array}$ & Inactive & {$[32,80]$} \\
\hline A. nitidum & Brazil & Trunk bark & $\mathrm{H}_{2} \mathrm{O}$ extract & In vivo/P. berghei & Inactive & [44] \\
\hline A. oblongum & Brazil & - & $\mathrm{EtOH}$ & $\begin{array}{l}\text { In vitro/P. falciparum } \\
\text { W2 and D6 }\end{array}$ & $\begin{array}{l}W 2: I_{50}=4742.5 \mathrm{ng} / \mathrm{mL} \\
D 6: I C_{50}=847.4 \mathrm{ng} / \mathrm{mL}\end{array}$ & [37] \\
\hline A. olivaceum & Brazil & - & $\begin{array}{l}\text { EtOH } \\
\text { Hexane-AcOEt }\end{array}$ & In vivo/P. berghei & Inactive & {$[67]$} \\
\hline A. olivaceum & Brazil & $\begin{array}{l}\text { Leaves, } \\
\text { trunk wood, } \\
\text { trunk bark }\end{array}$ & DCM and EtOH & $\begin{array}{l}\text { In vitro/P. falciparum } \\
\text { W2 and 3D7 }\end{array}$ & $\begin{array}{l}W 2: I_{50}=5.0 \text { to } 7 \mu \mathrm{g} / \mathrm{mL} \\
3 D 7: \mathrm{IC}_{50}=5.0 \text { to } 25.5 \mu \mathrm{g} / \mathrm{mL}\end{array}$ & [27] \\
\hline \multirow[t]{2}{*}{ A. olivaceum } & Brazil & - & Olivacine (4) & $\begin{array}{l}\text { In vitro/P. falciparum } \\
\text { 3D7 and K1 }\end{array}$ & $\begin{array}{l}\mathrm{K} 1: \mathrm{IC}_{50}=1.4 \mu \mathrm{M} \\
\text { 3D7: IC } 50=1.2 \mu \mathrm{M} \\
\text { Citotoxicity murine macrophages: } \\
\mathrm{CC}_{50}>4.1 \times 10^{2} \mu \mathrm{g} / \mathrm{mL} \\
\mathrm{SI}: \mathrm{K} 1>2.9 \times 10^{2} \\
3 \mathrm{D} 7>3.4 \times 10^{2}\end{array}$ & [48] \\
\hline & & & & In vivo/P. berghei & In vivo: active $(50 \mathrm{mg} / \mathrm{kg} /$ day $)$ & \\
\hline A. parvifolium & Brazil & Trunk bark & $\begin{array}{l}\text { EtOH extract, } \\
\text { Uleine (3) }\end{array}$ & $\begin{array}{l}\text { In vitro/P. falciparum } \\
\text { W2 and 3D7 } \\
\text { Uleine } \\
\text { W2 and 3D7 }\end{array}$ & $\begin{array}{l}W 2: I C_{50}=32.8 \mu \mathrm{g} / \mathrm{mL} \\
3 D 7: I C_{50}=20.5 \mu \mathrm{g} / \mathrm{mL} \\
W 2: I C_{50}=0.75 \mu \mathrm{g} / \mathrm{mL}(2.81 \mu \mathrm{M}) \\
3 D 7: I C_{50}=11.90 \mu \mathrm{gL}(32.69 \mu \mathrm{M})\end{array}$ & [27] \\
\hline A. polyneuron & Brazil & Trunk bark & Total alkaloids & In vitro/P. cathemerium & Active & [33] \\
\hline A. pyrifolium & Bolívia & Stem bark & $\mathrm{EtOH}-\mathrm{H}_{2} \mathrm{O}(7: 3)$ & $\begin{array}{l}\text { In vitro/P. falciparum } \\
\text { F32 strain } \\
\text { FBIT }\end{array}$ & Inactive & {$[40,81]$} \\
\hline A. pyrifolium & Bolivia & Stem bark & *Eight alkaloids & $\begin{array}{l}\text { In vitro/P. falciparum } \\
\text { FcM29 and Nigerian } \\
\text { strain }\end{array}$ & $\begin{array}{l}\mathrm{FCM} 29: \mathrm{IC}_{50}=3.2 \text { to } 28.5 \mu \mathrm{M} \\
\text { Nigerian strain: } \mathrm{IC}_{50}=5.1 \text { to } 22.6 \mu \mathrm{M}\end{array}$ & $\begin{array}{l}{[42]} \\
{[41]}\end{array}$ \\
\hline $\begin{array}{l}\text { A. quebracho- } \\
\text { blanco }\end{array}$ & Argentina & Trunk bark & Total alkaloids & Human & Active & {$[35,80]$} \\
\hline $\begin{array}{l}\text { A. quebracho- } \\
\text { blanco }\end{array}$ & Argentina & Trunk bark & $\mathrm{EtOH}$ & Human & Inactive & {$[34,80]$} \\
\hline $\begin{array}{l}\text { A. quebracho- } \\
\text { blanco }\end{array}$ & Bolívia & $\begin{array}{l}\text { Leaves, } \\
\text { trunk bark }\end{array}$ & EtOH- $\mathrm{H}_{2} \mathrm{O}(7: 3)$ & $\begin{array}{l}\text { In vitro/P. falciparum } \\
\text { F32 strain } \\
\text { FBIT }\end{array}$ & $\begin{array}{l}\text { Trunk bark extract } \\
\text { F32: } I C_{50}=3.9 \mu \mathrm{g} / \mathrm{mL} \\
\text { FBIT: } \mathrm{IC}_{50}=1.22 \mathrm{mg} / \mathrm{mL} \\
\text { Leaf extract: inactive }\end{array}$ & $\begin{array}{l}\text { [38] } \\
\text { continued }\end{array}$ \\
\hline
\end{tabular}




\begin{tabular}{|c|c|c|c|c|c|c|}
\hline $\begin{array}{l}\text { Aspidosperma } \\
\text { species }\end{array}$ & $\begin{array}{l}\text { Country of } \\
\text { collection }\end{array}$ & $\begin{array}{l}\text { Part of the } \\
\text { plant }\end{array}$ & $\begin{array}{l}\text { Extracts } \\
\text { Compounds }\end{array}$ & $\begin{array}{l}\text { Bioassay } \\
\text { Plasmodium species/ } \\
\text { strains }\end{array}$ & Results & $\begin{array}{l}\text { Refer- } \\
\text { ences }\end{array}$ \\
\hline A. ramiflorum & Brazil & $\begin{array}{l}\text { Leaves, } \\
\text { trunk wood, } \\
\text { trunk bark }\end{array}$ & DCM and EtOH & $\begin{array}{l}\text { In vitro/P. falciparum } \\
\text { W2 and 3D7 }\end{array}$ & $\begin{array}{l}W 2: I_{50}=19.7 \text { to } 36.5 \mu \mathrm{g} / \mathrm{mL} \\
3 D 7: I C_{50}=1.0 \text { to } 48.0 \mu \mathrm{g} / \mathrm{mL}\end{array}$ & [27] \\
\hline A. ramiflorum & Brazil & - & - & $\begin{array}{l}\text { In vitro/P. falciparum W1 } \\
\text { In vivo/P. berghei }\end{array}$ & $\begin{array}{l}\mathrm{W} 2: \mathrm{IC} 50=11 \text { to } 40 \mu \mathrm{g} / \mathrm{mL} \\
\text { In vivo: partial activity }\end{array}$ & {$[82]$} \\
\hline A. rigidum & $\begin{array}{l}\text { Peru } \\
\text { (Remo } \\
\text { caspi) }\end{array}$ & Trunk bark & $\mathrm{EtOH}$ & $\begin{array}{l}\text { In vitro/P. falciparum } \\
\text { 3D7 }\end{array}$ & $\begin{array}{l}\mathrm{IC}_{50}<10 \mu \mathrm{g} / \mathrm{mL} \\
\text { SI humam lymphocyte inhibition } 75 \%\end{array}$ & [39] \\
\hline A. spruceanum & Brazil & $\begin{array}{l}\text { Leaves, } \\
\text { trunk wood, } \\
\text { trunk bark }\end{array}$ & $\mathrm{DCM}$ and EtOH & $\begin{array}{l}\text { In vitro/P. falciparum } \\
\text { W2 and 3D7 }\end{array}$ & $\begin{array}{l}W 2: I C_{50}=<6.0 \text { to } 65.0 \mu \mathrm{g} / \mathrm{mL} \\
\text { 3D7: IC } 50=<6.0 \text { to }>100 \mu \mathrm{g} / \mathrm{mL}\end{array}$ & [27] \\
\hline A. tomentosum & Brazil & $\begin{array}{l}\text { Leaves, } \\
\text { trunk wood, } \\
\text { seeds, fruits }\end{array}$ & EtOH & $\begin{array}{l}\text { In vitro/P. falciparum } \\
\text { W2 and 3D7 }\end{array}$ & $\begin{array}{l}W 2: I_{50}=20.5 \text { to } 26.5 \mu \mathrm{g} / \mathrm{mL} \\
3 D 7: I C_{50}=3.0 \text { to } 38.5 \mu \mathrm{g} / \mathrm{mL}\end{array}$ & [27] \\
\hline A. ulei & Brazil & $\begin{array}{l}\text { Leaf, bark, } \\
\text { trunk wood, } \\
\text { root wood, } \\
\text { root bark }\end{array}$ & Five alkaloids** & $\begin{array}{l}\text { In vitro/P. falciparum } \\
\mathrm{K} 1\end{array}$ & $\begin{array}{l}\mathrm{K} 1: \mathrm{IC}_{50}=16.7 \text { to }>176.0 \mu \mathrm{M} \\
\text { Citotoxicity NIH } 3 \mathrm{~T} 3 \text { murine fibroblasts }\end{array}$ & [47] \\
\hline A. vargasii & Brazil & Trunk bark & Ellipticine (1) & In vitro/P. falciparum K1 & $\mathrm{IC}_{50}=0.073 \mu \mathrm{M}(0.018 \mu \mathrm{g} / \mathrm{mL})$ & [45] \\
\hline A. vargasii & Brazil & Bark & Ellipticine (1) & $\begin{array}{l}\text { In vitro/P. falciparum } \\
\text { 3D7 and K1 }\end{array}$ & $\begin{array}{l}\mathrm{K} 1: \mathrm{I} C_{50}=0.81 \mu \mathrm{M} \\
\text { 3D7: } \mathrm{IC}_{50}=0.35 \mu \mathrm{M} \\
\text { Citotoxicity murine macrophages: } \\
\mathrm{CC}_{50}>4.1 \times 10^{2} \mu \mathrm{g} / \mathrm{mL} \\
\mathrm{SI}: \mathrm{K} 1>5.0 \times 10^{2} \\
3 \mathrm{D} 7>1.2 \times 10^{3}\end{array}$ & [48] \\
\hline & & & & In vivo/P. berghei & In vivo: active $(50 \mathrm{mg} / \mathrm{kg} /$ day $)$ & \\
\hline
\end{tabular}

${ }^{*}$ Aspidospermidine (6), 10-methoxy-aspidospermidine (7), N-formil-aspidospermidine (8), vallesine (9), (-)- aspidospermine (10), demethoxyaspidospermine (11), palosine (12), haplocine (13). ** 20-epi-dasycarpidone (3), 3,4,5,6-tetradehydro- $\beta$-yohimbine, 20(E)-nor-subincanadine E, 19E-hunteracine, 12-hydroxy- $N$-acetyl-21( $N$ )-dehydroplumeran-18-oic acid

of plant species for antimalarial screening. Negative results were described for in vivo assays of $A$. nitidum crude extracts [32,44] ( Table 2). These results are surprising as A. nitidum is one of the most frequently cited Aspidosperma species of those used to treat malaria/fevers in Brazil. Therefore, this species deserves further investigation with a focus on the alkaloids.

Interestingly, a strong interest in Aspidosperma species has resumed in the past two decades, and several species whose phytochemistry was intensively investigated during 1960-1980 have been reexamined, leading to the isolation of several known indole alkaloids. Several of these alkaloids have been evaluated for various biological/pharmacological effects [30]. A total of 20 Aspidosperma alkaloids have been assayed for antimalarial activity and have been isolated by Brazilian researchers, one from $A$. desmanthum [45], one from $A$. vargasii [45], one from $A$. parvifolium $[27,46]$, five from A. ulei [47], and one from A. olivaceum [48]; additionally, a French-Bolivian group has evaluated 11 alkaloids from $A$. megalocarpon and A. pyrifolium [41].

Ellipticine (1) and aspidocarpine (2) ( $\bullet$ Fig. 1) have been isolated from the trunk bark of $A$. vargasii and $A$. desmanthum, respectively. Both of these species have been collected at the Ducke Reserve in Manaus, Amazonas state, Brazil, and they have shown remarkable in vitro activity against the multidrug-resistant K1 strain of $P$. falciparum ( $\mathrm{IC}_{50}=73 \mathrm{nM}$ and $19 \mathrm{nM}$, respectively) [45]. Ellipticine (1), a pyridocarbazol alkaloid, was originally isolated from Ochrosia elliptica (an Australian evergreen shrub) and occurs in other genera of the family Apocynaceae, such as Aspidosperma and Bleekeria [49-51]. Ellipticine (1) and several related syn- thetic derivatives are highly cytotoxic to human cancer cell lines, and elliptinium acetate (9-OH-NME, Celliptium ${ }^{\circledR}$ ) is an antineoplastic agent currently used in the treatment of metastatic breast cancer [49-51]. Recently, ellipticine (1) and olivacine (5) ( $\bullet$ Fig. 1) were assayed against P. falciparum strains K1 and 3D7. Olivacine disclosed lower in vitro antimalarial activity than ellipticine, and low cytoxicity for both agents was observed against murine macrophages. Ellipticine was also more active than olivacine in an evaluation of the effects in P. berghei-infected mice. Remarkably, $100 \%$ parasitemia suppression was observed at an oral dose of $50 \mathrm{mg} / \mathrm{kg} /$ day (four days), and no mortality or other signs of toxicity were reported [48]. Ellipticine (1) and its derivatives were described as the most active compounds, with $\mathrm{IC}_{50}$ values $<1 \mu \mathrm{g} / \mathrm{mL}$ (range 0.08 to $0.47 \mu \mathrm{g} / \mathrm{mL}$ ), in a series of 184 randomly selected compounds belonging to several classes of natural products, either from plants or from marine organisms, or prepared as intermediates during the synthesis or semisynthesis of isolated products that were assayed against the P. falciparum FcM29 strain [52].

A total of 12 indole alkaloids were recently isolated from the EtOH extracts of various parts of A. ulei Markgr. Only five of these alkaloids were assayed for antimalarial activity against the multidrug-resistant K1 strain of $P$. falciparum. 20-Epi-dasycarpidone (3) ( Fig. 1) showed moderate activity ( $\mathrm{IC}_{50} 4.5 \mu \mathrm{g} / \mathrm{mL}$, $16.7 \mu \mathrm{M})$, whereas 3,4,5,6-tetradehydro- $\beta$-yohimbine, $19 E$-hunteracine, 20(E)-nor-subincanadine E, and 12-hydroxy- $N$-acetyl-21 $(N)$-dehydroplumeran-18-oic acid were inactive. Two of the known alkaloids isolated, uleine and olivacine, have previously 
<smiles>Cc1c2ccncc2c(C)c2c1[nH]c1ccccc12</smiles>

(1) Ellipticine

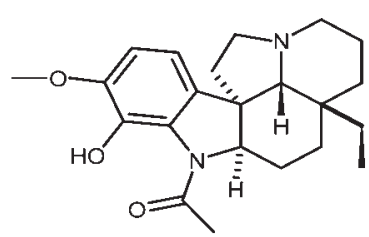

(2) Aspidocarpine

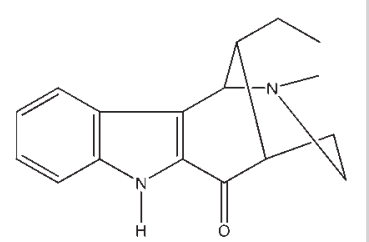

(3) 20-Epi-dasycarpidone
Fig. 1 Chemical structures of antimalarial alkaloids from Aspidosperma species: ellipticine from A. vargasii [45], aspidocarpine from $A$. desmanthum [45], uleine from A. parvifolium [46], olivacine from A. olivaceum [47] and 20-Epi-dasycarpidone from A. ulei [47].<smiles>C=C1c2c(c3ccccc3n2I)C(CC)N1C</smiles>

(4) Uleine<smiles>Cc1nccc2c(C)c3[nH]c4ccccc4c3cc12</smiles>

(5) Olivacine been shown to be active $[46,48]$. The compounds were evaluated against NIH3T3 murine fibroblasts and showed no cytotoxicity up to a concentration of $50 \mu \mathrm{g} / \mathrm{mL}$ [47].

Eleven known aspidospermane alkaloids ( $\bullet$ Fig. 2) were isolated from A. pyrifolium and A. megalocarpon, both collected in Bolivia $[42,53]$ and both also occurring in Brazil [24-26]. Three of the active compounds, aspidospermine (6), 10-methoxy-aspidospermidine (7), and $N$-formyl-aspidospermine (8) (๑ Fig.2), were shown to be less cytotoxic to NIH 3 T3 cells (human fibroblasts), with calculated SI values of 22.7, 15.6, and 8.3, respectively [41]. The in vitro antiplasmodial activity of $A$. megalocarpon bark extract against D2 and F32 strains of $P$. falciparum has been reported; subsequently, a very low $\mathrm{SI}(<1)$ was reported in relation to cytotoxicity assays in U-937 human promonocytic cells [36]. Uleine (4) (๑ Fig. 1) was first isolated from A. ulei in 1959 [54,55]. It is frequently found in species, e.g., A. australe, A. dasycarpon, A. eburneum, A. excelsum, A. formosanum, A. gilbertii, A. gomezianum, A. multiflorum, A. nigricans, A. olivaceum, and A. parvifolium [29], as well as in other apocynaceous representatives such as $\mathrm{Hi}$ mathanthus lancifolius [56].

A recent phytochemical reinvestigation of $A$. parvifolium trunk bark afforded four known indole alkaloids, uleine (4) (๑ Fig. 1), epi-uleine, apparicine, and $N$-demethyluleine [57]. A bioguided fractionation of the trunk bark alkaloidal fraction demonstrated the in vitro activity of uleine against chloroquine-resistant $P$. falciparum (W2), with an $\mathrm{IC}_{50}$ at the ng level, at least against the W2 clone (Table 2) $[27,46]$. The antiplasmodial activity of uleine (4), an aspidospermane indole alkaloid, may be related to the inhibition of heme polymerization to give hemozoin, as demonstrated by its in vivo effect in the food vacuole of chloroquine-resistant $P$. falciparum (W2 clone) monitored by confocal microscopy [46].

Uleine (4) ( Fig. 1) has been evaluated for cytotoxicity against the NCI 60 cancer cell line panel and was inactive in all of them [58], reinforcing its high potential as a leading antimalarial compound.

In summary, crude extracts, alkaloidal extracts, and isolated alkaloids from 19 Aspidosperma species have been evaluated for antimalarial activity, and positive results have been observed for most of them. These results disclose the high potential of Aspidosperma species as sources of antimalarial alkaloids. A substantial literature search, approximately 1031 articles, describes the antiplasmodial activity of plant extracts, but very few of these re-

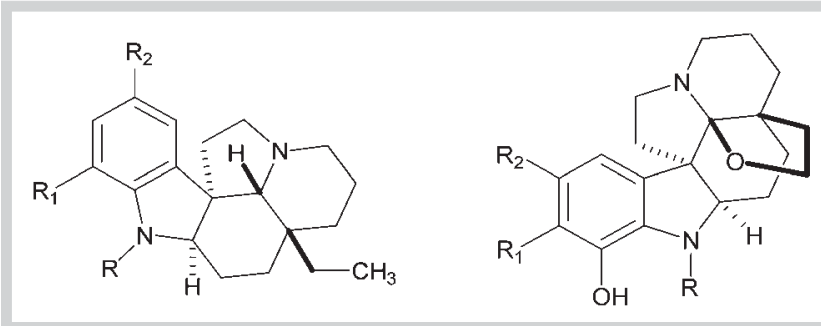

Fig. 2 Antimalarial alkaloids from A. megalocarpon and A. pyrifolium [41].

ports include the effect of pure constituents [14], a requirement for the development of new drugs. The development of new drugs is lengthy and extremely costly. However, if effective, safe, and locally produced phytomedicines are the goal, a more direct and less expensive route might certainly be pursued $[5,59,60]$.

\section{Conclusions}

$\nabla$

Our literature survey has identified 24 Aspidosperma species reported to be used to treat malaria/fevers. Of these, a total of 19 species have had extracts and/or isolated alkaloids evaluated for antimalarial activity by different assays, showing positive results. Only 20 of more than 200 known indole alkaloids from Aspidosperma species have been assayed for antimalarial activity, and variable levels of parasite inhibition have been observed. Among the assayed Aspidosperma alkaloids, uleine (4) ( $\bullet$ Fig. 1) appears the most promising as an antimalarial because it has shown good in vitro activity $[27,46]$ and no cytotoxicity in several human cancer cell lines [58]. These findings have motivated a patent in Brazil [61]. However, recent results on the in vivo evaluation of ellipticine (1) ( Fig. 1) appear to place this alkaloid in the leading position among natural antimalarials because it has, remarkably, shown $100 \%$ parasitemia suppression at an oral dose of $50 \mathrm{mg} /$ $\mathrm{kg} /$ day and no signs of mortality or toxicity [48]. Our review also demonstrates a need for molecular genetic studies to facilitate the identification and the differentiation of Aspidosperma species and, therefore, to clarify the controversial question of synonyms. The high chemical diversity of alkaloids from Aspidosperma species and the small number of these alkaloids that have been as- 
sayed for antimalarial activity coupled with the traditional use of several species of this taxon to treat malaria in Brazil as well as in other Meso- and South American countries make further investigations of the plants in this genus of great interest in the quest for natural antimalarial products. There is a need to isolate plant constituents to evaluate their pharmacological profile, to further pursue studies of synergistic effects, and to make these constituents available for use as markers in the standardization of extracts to be used in the development of effective, safe, and lowcost phytomedicines. These phytomedicines would then be available to Amazonian people, who inhabit the region where many of the Aspidosperma species occur and that have the highest incidence of malaria in Meso- and South America.

\section{Acknowledgements}

We thank CNPq and FAPEMIG for financial support (PRONEX CNPq Process 555655/2009-1 and FAPEMIG Process CDS APQ 01129-10) and a doctoral fellowship (CNPq to RCP). Additionally, we thank Dr. Luzia H. Carvalho, CPqRR/FIOCRUZ, Belo Horizonte, Brazil, for helpful discussions.

\section{Conflict of Interest}

$\nabla$

The authors declare that they do not have any conflict of interest in reference to the content of this article.

\section{References}

1 World Health Organization. World Malaria Report 2009. Available at http://whqlibdoc.who.int/publications/2009/9789241563901_eng. pdf. Accessed November 25, 2009.

2 World Health Organization. World Malaria Report 2008. Available at http://whqlibdoc.who.int/publications/2008/9789241563697_eng. pdf. Accessed November 22, 2009.

3 Oliveira-Ferreira J, Lacerda MVG, Brasil P, Ladislau JLB, Tauil PL, DanielRibeiro CT. Malaria in Brazil: an overview. Malar J 2010; 9: 115-130

4 World Health Organization. World Malaria Report 2011. Available at http://www.who.int/malaria/world_malaria_report_2011/ 9789241564403_eng.pdf. Accessed January 13, 2011.

5 Oliveira AB, Dolabela MF, Braga FC, Jacome RL, Varotti FP, Povoa MM. Plant-derived antimalarial agents: new leads and efficient phythomedicines. Part I. Alkaloids. An Acad Bras Cienc 2009; 81: 715-740

6 Castellanos JRG, Prieto JM, Heinrich M. Red Lapacho (Tabebuia impetiginosa)-a global ethnopharmacological commodity? J Ethnopharmacol 2009; 121: 1-13

7 Newman DJ, Cragg GM. Natural products as sources of new drugs over the last 25 years. J Nat Prod 2007; 70: 461-477

8 Ginsburg H, Deharo E. A call for using natural compounds in the development of new antimalarial treatments - an introduction. Malar J 2011; 10 (Suppl. 1): S1

9 Batista R, Junior AJS, Oliveira AB. Plant-derived antimalarial agents: new leads and efficient phytomedicines. Part II. Non-alkaloidal natural products. Molecules 2009; 14: 3037-3072

10 Verpoorte R, Choi Y, Mustafa N, Kim H. Metabolomics: back to basics. Phytochem Rev 2008; 7: 525-537

11 Wagner $H$. Natural products chemistry and phytomedicine research in the new millennium: new developments and challenges. Arkivoc 2004; VII: 277-284

12 Ulrich-Merzenich G, Zeitler H, Jobst D, Panek D, Vetter H, Wagner H. Application of the "-Omic-" technologies in phytomedicine. Phytomedicine 2007; 14: 70-82

13 Verpoorte R, Choi YH, Kim HK. Ethnopharmacology and systems biology: a perfect holistic match. J Ethnopharmacol 2005; 100: 53-56

14 Deharo E, Ginsburg H. Analysis of additivity and synergism in the antiplasmodial effect of purified compounds from plant extracts. Malar J 2011; 10 (Suppl. 1): S5
15 Lorenzi H. Árvores Brasileiras: manual de identificação e cultivo de plantas arbóreas nativas do Brasil. 2nd edition. São Paulo: Plantarum; 1998: 365

16 Perez-Arbeláez E. Plantas útiles de Colombia. 5th edition. Bogotá: Fondo Fen Colombia; 1996: 831

17 Pio-Corrêa M. Dicionário de plantas úteis do Brasil e das exóticas cultivadas. Rio de Janeiro: Ministério da Agricultura; 1978: 687

18 Rizzini CT. Árvores e madeiras úteis do Brasil: manual de dendrologia brasileira. 2nd edition. São Paulo: E. Blücher; 2000: 312

19 Schultes RE, RaffaufRF. The healing forest: medicinal and toxic plants of the northwest Amazonia. 1st edtion. Portland: Dioscorides Press; 2003: 500

20 Koch I, Rapini A, Kinoshita LS, Simões AO, Spina AP. Apocynaceae. Lista de Espécies da Flora do Brasil. Jardim Botânico do Rio de Janeiro. Available at http://reflora.jbrj.gov.br/jabot/floradobrasil/FB21892. Accessed December 8, 2013.

21 Sennblad B, Bremer B. Classification of Apocynaceae s.l. according to a new approach combining Linnaean and phylogenetic taxonomy. Syst Biol 2002; 51: 389-409

22 Woodson RE. Studies in the Apocynaceae. VIII. An Interim Revision of the Genus Aspidosperma Mart. \& Zucc. Ann Mo Bot Gard 1951; 38 : 119-206

23 Marcondes-Ferreira $W$, Kinoshita $L$. Uma nova divisão infragenerica para Aspidosperma Mart. (Apocynaceae).[A new infrageneric division on Aspidosperma Mart. (Apocynaceae)]. Rev Brasil Bot 1996; 19: 203 214

24 Lista de Espécies da Flora do Brasil. Available at http://floradobrasil. jbrj.gov.br/jabot/listaBrasil/PrincipalUC/PrincipalUC.do. Accessed November $29,2011$.

25 Tropicos. Missouri Botanical Garden - Tropicos Specimen Data Base. Available at http://www.tropicos.org/NameSearch.aspx?name= Aspidosperma\&common name $=$. Accessed November 30, 2011.

26 The Plant List. (2010). Version 1. Available at http://www.theplantlist org/tpl/search?q=Aspidosperma. Accessed November 29, 2011.

27 Dolabela MF. Atividade antiplasmódica e citotoxicidade de Esenbeckia febrifuga (A.St-Hil.) Juss. ex Mart. (Rutaceae) e de espécies do gênero Aspidosperma (Apocynaceae) [dissertation]. Belo Horizonte: Universidade Federal de Minas Gerais; 2007

28 Mesquita M, Grellier P, Mambu L, De Paula J, Espindola L. In vitro antiplasmodial activity of Brazilian Cerrado plants used as traditional remedies. J Ethnopharmacol 2007; 110: 165-170

29 Pereira MM, Jácome R, Alcântara A, Alves RB, Raslan DS. Alcalóides indólicos isolados de espécies do gênero Aspidosperma (Apocynaceae). Quim Nova 2007; 30: 970-983

30 Oliveira V, Freitas M, Mathias L, Braz-Filho R, Vieira I. Atividade biológica e alcalóides indólicos do gênero Aspidosperma (Apocynaceae): uma revisão. Rev Bras Pl Med 2009; 11: 92-99

31 Trager $W$, Jensen JB. Human malaria parasites in continuous culture. Science 1976; 193: 673-675

32 Becker ER. Report on 35 drugs and three plant materials tested against Plasmodium lophurae in the white Pekin duck. Iowa State Coll J Sci 1949; 23: 189-194

33 Wasicky R, Unti O, Barbieri E. Quinine and alkaloids in Brazil. An Fac Farm Odontol Univ São Paulo 1942; 3: 137

34 Dominguez J. Malaria treatment with Aspidosperma quebracho blanco. Rev Farm (Buenos Aires) 1932; 73: 82

35 Loizaga N, Sagastume L. Malaria treatment with Quechuol-Dominguez. Sem Med Buenos Aires 1935; 2: 562-566

36 Weniger B, Robledo S, Arango GJ, Deharo E, Aragon R, Munoz V, Callapa J, Lobstein A, Anton R. Antiprotozoal activities of Colombian plants. J Ethnopharmacol 2001; 78: 193-200

37 Cabral JA, McChesney JD, Milhous WK. A new antimalarial quassinoid from Simaba guianensis. J Nat Prod 1993; 56: 1954-1961

38 Bourdy G, Oporto P, Gimenez A, Deharo E. A search for natural bioactive compounds in Bolivia through a multidisciplinary approach. Part VI. Evaluation of the antimalarial activity of plants used by Isoceno-Guarani Indians. J Ethnopharmacol 2004; 93: 269-277

39 Kvist LP, Christensen SB, Rasmussen HB, Mejia K, Gonzalez A. Identification and evaluation of Peruvian plants used to treat malaria and leishmaniasis. J Ethnopharmacol 2006; 106: 390-402

40 Brandão MG, Grandi TS, Rocha EM, Sawyer DR, Krettli AU. Survey of medicinal plants used as antimalarials in the Amazon. J Ethnopharmacol 1992; 36: 175-182 
41 Mitaine-Offer AC, Sauvain M, Valentin A, Callapa J, Mallie M, ZechesHanrot M. Antiplasmodial activity of Aspidosperma indole alkaloids. Phytomedicine 2002; 9: 142-145

42 Mitaine AC, Mesbah K, Richard B, Petermann C, Arrazola S, Moretti C, Zeches-Hanrot M, Men-Olivier LL. Alkaloids from Aspidosperma species from Bolivia. Planta Med 1996; 62: 458-461

43 Dolabela MF, Oliveira SG, Peres JM, Nascimento JM, Póvoa MM, Oliveira $A B$. In vitro antimalarial activity of six Aspidosperma species from the state of Minas Gerais (Brazil). An Acad Bras Cienc 2012; 84: 899-910

44 Carvalho LH, Brandão MG, Santos-Filho D, Lopes JL, Krettli AU. Antimalarial activity of crude extracts from Brazilian plants studied in vivo in Plasmodium berghei-infected mice and in vitro against Plasmodium falciparum in culture. Braz J Med Biol Res 1991; 24: 1113-1123

45 Andrade-Neto VF, Pohlit AM, Pinto AC, Silva EC, Nogueira KL, Melo MR, Henrique MC, Amorim RC, Silva LF, Costa MR, Nunomura RC, Nunomura $S M$, Alecrim WD, Alecrim MG, Chaves FC, Vieira PP. In vitro inhibition of Plasmodium falciparum by substances isolated from Amazonian antimalarial plants. Mem Inst Oswaldo Cruz 2007; 102: 359-365

46 Oliveira AB, Dolabela MF, Póvoa MM, Santos CAM, Varotti FP. Antimalarial activity of ulein and proof of its action on the Plasmodium falciparum digestive vacuole. Mal J 2010; 9 (Suppl. 2): 09

47 Torres ZES, Silveira ER, Rocha e Silva LF, Lima ES, Vasconcellos MC, Andrade Uchoa DE, Filho RB, Pohlit AM. Chemical composition of Aspidosperma ulei Markgr. and antiplasmodial activity of selected indole alkaloids. Molecules 2013; 18: 6281-6297

48 Rocha e Silva LF, Montoia A, Amorim RC, Melo MR, Henrique MC, Nunomura SM, Costa MR, Andrade Neto VF, Costa DS, Dantas G, Lavrado J, Moreira R, Paulo A, Pinto AC, Tadei WP, Zacardi RS, Eberlin MN, Pohlit $A M L F$. Comparative in vitro and in vivo antimalarial activity of the indole alkaloids ellipticine, olivacine, cryptolepine and a synthetic cryptolepine analog. Phytomedicine 2012; 20: 71-76

49 Cirino JJV, Belletato P, Dantas SO, Ribeiro L, Ferreira GR, Santos HF. Estrutura e propriedades de elipticinas. Quim Nova 2005; 28: 30-36

50 Cragg GM, Newman DJ. Plants as a source of anti-cancer agents. JEthnopharmacol 2005; 100: 72-79

51 Mans DR, da Rocha AB, Schwartsmann G. Anti-cancer drug discovery and development in Brazil: targeted plant collection as a rational strategy to acquire candidate anti-cancer compounds. Oncologist 2000; 5: 185-198

52 Passemar C, Salery M, Soh PN, Linas MD, Ahond A, Poupat C, Benoit-Vical $F$. Indole and aminoimidazole moieties appear as key structural units in antiplasmodial molecules. Phytomedicine 2011; 18: 1118-1125

53 Mitaine AC, Weniger B, Sauvain M, Lucumi E, Aragon R, Zeches-Hanrot M. Indole alkaloids from the trunk bark of Aspidosperma megalocarpon. Planta Med 1998; 64: 487

54 Büchi G, Warnhoff E. The structure of uleine. J Am Chem Soc 1959; 81: 4433-4434

55 Joule J. The uleine-ellipticine-vallesamine group. In: Saxton JE, editor. The chemistry of heterocyclic compounds, indoles: the monoterpenoid indole alkaloids. 1st edition. Cleveland: John Wiley \& Sons; 2009: 886

56 Nardin JM, de Souza WM, Lopes JF, Florao A, de Moraes Santos CA, Weffort-Santos AM. Effects of Himatanthus lancifolius on human leukocyte chemotaxis and their adhesion to integrins. Planta Med 2008; 74: $1253-1258$

57 Jácome R, Oliveira A, Raslan DS, Wagner H. Estudo químico e perfil cromatográfico das cascas de Aspidosperma parvifolium A. DC. ("pau-pereira"). Quim Nova 2004; 27: 897-900

58 PubChem Substance. Uleine-Substance Summary. Available at http:// pubchem.ncbi.nlm.nih.gov/summary/summary.cgi?sid=115962. Accessed August 11, 2013.

59 Willcox ML, Bodeker G. Traditional herbal medicines for malaria. BMJ 2004; 329: 1156-1159

60 Wright CW. Recent developments in research on terrestrial plants used for the treatment of malaria. Nat Prod Rep 2010; 27: 961-968

61 Oliveira $A B$, Dolabela MF, Rocha FD, Gomes FMA, Neiva RMT, Jácome RLRP. Extrato e fração padronizados de cascas de Aspidosperma parvifolium e/ou uleina e sua composição farmacêutica. Filing date: Dec 23,
2009. PI0905584-3. Assignee: Universidade Federal de Minas Gerais UFMG. Belo Horizonte, MG, Brazil

62 Araújo jr. JX, Antheaume C, Trindade RCP, Schmitt M, Bourguignon IJ. Sant'Ana AEG. Isolation and characterisation of the monoterpenoid indole alkaloids of Aspidosperma pyrifolium. Phytochem Rev 2007; 6: 183-188

63 Grenand P, Moretti C, Jacquemin H, Prévost M. Pharmacopées traditionnelles en Guyane Créoles, Polikur, Wayapi. 1st edition. Montpellier: IRD Édtions; 2006: 816

64 Barbosa WLR, Tavares ICC, Soares DC. Alcaloides de Aspidosperma auriculatum Standl. Braz J Pharmacogn 2003; 13: 6-8

65 Pittier $\mathrm{H}$. Manual de las plantas usuales de Venezuela y su suplemento. 2nd edition. Caracas: Fundación Eugenio Mendoza; 1978: 620

66 Quignard ELJ, Martin Pohlit A, Nunomura SM, Da Silva Pinto AC, Mustafa dos Santos EV, Reis de Morais SK, Alecrim AM, Da Silva Pedroso AC, Brito Cyrino BR, Melo CS. Screening of plants found in Amazonas state for lethality towards brine shrimp Artemia franciscana. Acta Amazonica 2003; 33: 93-104

67 Brandão MGL, Botelho MGA, Krettli AU. Quimioterapia experimental antimalárica com produtos naturais: I. Uma abordagem mais racional?/Experimental antimalarial chemotherapy with natural products: I. A more rational approach? Cienc Cult 1985; 37: 1152-1163

68 Milliken W. Traditional anti-malarial medicine in Roraima, Brazil. Econ Bot 1997; 51: 212-237

69 Mejía CK, Rengifo SE. Plantas medicinales de uso popular en la Amazonía Peruana. 1st edition. Lima: Tarea Asociación Gráfica Educativa; 1995: 286

70 Pérez $D$. Etnobotánica medicinal y biocidas para malaria en la región Ucayali. Folia Amazónica 2002; 13: 2

71 Duke JA, Vasquez R. Amazonian ethnobotanical dictionary. 1st edition. Florida: CRC Press; 1994: 224

72 Milliken $W$, Albert $B$. The use of medicinal plants by the Yanomami Indians of Brazil. Econ Bot 1996; 50: 10-25

73 DeFilipps RA, Maina SL, Crepin J. Medicinal Plants of the Guianas (Guyana, Surinam, French Guiana). Part 1 Acanthaceae - Combretaceae. Avaliable at http://botany.si.edu/bdg/medicinal/Medicinal_plants_master. pdf. Accessed July 28, 2011.

74 Almeida MR, Lima JA, dos Santos NP, Pinto AC. Pereirina: o primeiro alcalóide isolado no Brasil? Braz J Pharmacogn 2009; 19: 942-952

75 Botsaris AS. Plants used traditionally to treat malaria in Brazil: the archives of Flora Medicinal. J Ethnobiol Ethnomed 2007; 3: 18-19

76 Milliken W, Albert B. The use of medicinal plants by the Yanomami Indians of Brazil, Part II. Econ Bot 1997; 51: 264-278

77 Del Vitto LA, Petenatti E, Petenatti M. Recursos herbolarios de San Luis (República Argentina). Primera parte: Plantas nativas. Multequina 1997; 6: 49-66

78 Freitas ADD. Aspectos tecnológicos e morfoanatômicos de sementes maduras, plântulas e plantas jovens de Aspidosperma spruceanum Benth. Ex Mull. Arg. (Apocynaceae) [dissertation]. Belém: Universidade Federal Rural da Amazônia e Museu Paraense Emílio Goeldi; 2008

79 Di Stasi LC. Plantas medicinais na Amazônia e na Mata Atlântica. 2nd edition. São Paulo: UNESP; 2002: 592

80 Mariath IR, Falcão HS, Barbosa-Filho JM, Sousa LCF, Tomaz ACA, Batista LM, Diniz MFFM, Athayde-Filho PF, Tavares JF, Silva MS. Plants of the American continent with antimalarial activity. Braz J Pharmacogn 2009; 19: 158-191

81 Muñoz V, Sauvain M, Bourdy G, Callapa J, Bergeron S, Rojas I, Bravo JA, Balderrama L, Ortiz B, Gimenez A, Deharo E. A search for natural bioactive compounds in Bolivia through a multidisciplinary approach. Part I. Evaluation of the antimalarial activity of plants used by the Chacobo Indians. J Ethnopharmacol 2000; 69: 127-137

82 Krettli LG, de Paula RC, Varotti FP, Pereira MM, Oliveira AJB, Krettli AU. Antimalarial activity of extracts from Aspidosperma plants tested in vitro against Plasmodium falciparum drug resistant parasites and in animal models. XXIV Meeting of the SBPz. Águas de Lindóia, São Paulo: Brazilian Society of Protozoology; 2008 